\title{
ON ALGEBRAS OF CONTINUOUS FUNCTIONS
}

\author{
JOHN WERMER ${ }^{1}$
}

Let $C$ denote the algebra of functions continuous on the unit circle. With norm $\|f\|=\sup _{; \lambda \mid=1}|f(\lambda)|, C$ is a Banach algebra. Let $A$ denote the set of all $f$ in $C$ which are boundary values of functions analytic in $|z|<1$ and continuous in $|z| \leqq 1 . A$ is then a closed subalgebra of $C$, and by known results $A$ consists of those and only those $f$ in $C$ for which $\int_{|\lambda|-1} f(\lambda) \lambda^{n} d \lambda=0, n \geqq 0$.

In [1] the question was raised whether if $\phi(\lambda)$ is any function in $C$ and not in $A$, the closed algebra generated by $\phi$ and $A$ is all of $C$. It was shown in [1] that if $\phi$ is real or if $\phi$ satisfies a Lipschitz condition, then the algebra generated by $A$ and $\phi$ does equal $C$. In the following theorem the question is answered in the affirmative.

Theorem 1. If $A^{\prime}$ is any closed subalgebra of $C$ which includes $A$, then either $A^{\prime}=A$ or $A^{\prime}=C$.

Our proof does not make use of the results of [1]. Our chief tool is the following known theorem (see [2, pp. 19-21;3, p. 162]). Let $d \mu(\lambda)$ be a complex-valued measure on the circle such that $\int_{|\lambda|-1} \lambda^{n} d \mu(\lambda)=0$, $n \geqq 0$. Then there exists a function $h(z)$ analytic in $|z|<1$ with the following properties: $h\left(e^{i \theta}\right)=\lim _{r-1} h\left(r e^{i \theta}\right)$ exists for almost all $\theta$ and is summable on $[0,2 \pi]$ and $\lim _{r=1} \int_{0}^{2 \pi}\left|h\left(r e^{i \theta}\right)-h\left(e^{i \theta}\right)\right| d \theta=0$ and $d \mu(\lambda)=h(\lambda) d \lambda$, i.e. if $f$ is in $C, \int_{|\lambda|-1} f(\lambda) d \mu(\lambda)=\int_{|\lambda|=1} f(\lambda) h(\lambda) d \lambda$. For a given $\theta$, moreover, $h(z)$ approaches $h\left(e^{i \theta}\right)$ along any nontangential path.

Proof of Theorem 1. Suppose $A^{\prime} \neq C$. Let $\phi(\lambda)$ be any function in $A^{\prime}$. We claim that $\phi$ is in $A$.

For since $A^{\prime}$ is a proper closed subspace of $C$, there exists a linear functional on $C$ which is not null but which annihilates every $f$ in $A^{\prime}$ and which hence annihilates every function $\lambda^{n} \phi^{m}(\lambda)$ with $n, m \geqq 0$. By the representation of linear functionals on $C$, there thus exists a complex measure $d \mu(\lambda)$ with

$$
\int_{|\lambda|=1} \lambda^{n} \phi^{m}(\lambda) d \mu(\lambda)=0, \quad n, m \geqq 0 .
$$

Presented to the Society, February 28, 1953; received by the editors January 13 , 1953 and, in revised form, March 16, 1953.

1 The author is a fellow of the National Science Foundation. The author wishes to thank Professor C. Rickart and Professor S. Kakutani for some interesting conversations about the subject of this paper. 
In particular, for $m=0$, we get $\int_{|\lambda|-1} \lambda^{n} d \mu(\lambda)=0$. As we noted, this implies the existence of an analytic function $h(z)$ with $d \mu(\lambda)=h(\lambda) d \lambda$. Fix now $m$ and consider the measure $\phi^{m}(\lambda) h(\lambda) d \lambda$ on $|\lambda|=1$. This measure annihilates $\lambda^{n}$ for $n \geqq 0$ and by the same theorem there exists an analytic function $\gamma_{m}(z)$ with $\phi^{m}(\lambda) h(\lambda)=\gamma_{m}(\lambda)$.

Set now $\gamma_{1}(\lambda)=g(\lambda)$. Then $g^{m}(\lambda)=\phi^{m}(\lambda) h^{m}(\lambda)=h^{m-1}(\lambda) \gamma_{m}(\lambda)$ for $|\lambda|=1$. But $h^{m-1} \cdot \gamma_{m}$ and $g^{m}$ are analytic functions and since their (nontangential) boundary values are equal almost everywhere, the functions themselves are equal, i.e. $g^{m}(z)=h^{m-1}(z) \gamma_{m}(z)$ for $|z|<1$. The last statement follows at once from a theorem of Priwaloff [4] which asserts that if an analytic function has nontangential boundary values on $|\lambda|=1$ vanishing on a set of positive measure, then the function vanishes identically.

We now assert that if $h$ has a zero of order $p$ at $\alpha,|\alpha|<1$, then $g$ has at $\alpha$ a zero of order $q \geqq p$. For since $g^{m}(z)=h^{m-1}(z) \gamma_{m}(z)$ and $\gamma_{m}$ is analytic at $\alpha$, we have $m q \geqq(m-1) p$. Since this holds for arbitrarily large $m$, we get $q \geqq p$ as asserted. The function $g(z) / h(z)$ is then analytic in $|z|<1$. Also, this function has $\phi\left(e^{i \theta}\right)$ as boundary value a.e. Thus for any $\phi$ in $A^{\prime}$ there exists a function $\Phi(z)$ analytic in $|z|<1$ and taking $\phi\left(e^{i \theta}\right)$ as (nontangential) boundary value a.e. By Priwaloff's theorem, then, $\phi$ determines $\Phi$ uniquely. If $\phi_{1}, \phi_{2} \in A^{\prime}$ and $\Phi_{1}, \Phi_{2}$ take boundary values $\phi_{1}$ and $\phi_{2}$ respectively, then $\Phi_{1} \cdot \Phi_{2}$ is the unique analytic function with boundary value $\phi_{1} \cdot \phi_{2}$, and similarly for $\Phi_{1}+\Phi_{2}$. Fix now $z_{0},\left|z_{0}\right|<1$. The mapping $\phi \rightarrow \Phi\left(z_{0}\right)$ is thus a multiplicative linear functional defined on the Banach algebra $A^{\prime}$. Hence $\left|\Phi\left(z_{0}\right)\right| \leqq\|\phi\|$. Since $z_{0}$ was arbitrary, each $\Phi(z)$ is bounded in $|z|<1$.

Take now any $\phi$ in $A^{\prime}$ and let $\Phi$ be the corresponding analytic function. Then the functions $\Phi_{r}(\theta)=\Phi\left(r e^{i \theta}\right)$ are uniformly bounded as $r$ approaches 1. Also $\phi\left(e^{i \theta}\right)=\lim _{r=1} \Phi_{r}(\theta)$ a.e. Hence $\int_{|\lambda|-1} \lambda^{n} \phi(\lambda) d \lambda$ $=\lim _{r-1} \int_{|\lambda|-r} \lambda^{n} \Phi(\lambda) d \lambda=0, n \geqq 0$.

Hence $\phi$ is in $A$, and the theorem is proved.

It is of interest to note the following concerning the proof we have given: In order that a function $\phi(\lambda)$ in $C$ belong to $A$ it is not suffcient that there exist a function $\Phi(z)$ analytic in $|z|<1$ and with nontangential boundary values equal to $\phi\left(e^{i \theta}\right)$ a.e. Let $\Phi(z)$ $=(1-z) e^{(1+z) /(1-z)}$. Then $\lim _{z \rightarrow e^{i \theta} \theta} \Phi(z)=\left(1-e^{i \theta}\right) e^{i \cot (\theta / 2)}=\phi(\theta)$ a.e. Now $\phi(\theta)$ is in $C$, and $\Phi(z)$ is even the quotient of two bounded analytic functions. Still $\phi(\theta)$ is not in $A$.

THEOREM 2. Let $B$ be any closed subalgebra of $C$ which contains one function $\phi(\lambda)$ such that $\phi\left(\lambda_{1}\right)=\phi\left(\lambda_{2}\right)$ implies $\lambda_{1}=\lambda_{2}$. Then if $B^{\prime}$ is any closed subalgebra of $C$ which includes $B$, either $B^{\prime}=B$ or $B^{\prime}=C$. 
Proof. Let $\Gamma$ be the simple closed Jordan curve which is the image of the unit circle under the function $\phi(\lambda)$. Let $\gamma(z)$ be the function which maps the interior of the unit circle conformally on the interior of $\Gamma$. Then $\gamma$ is continuous and one-to-one up to the boundary and so the map: $\lambda$ into $\gamma(\lambda)$ is a homeomorphism of the circle $|\lambda|=1$ on $\Gamma$. Let us now define the following mapping $\tau$ of $C$ into $C$ : If $f(\lambda)$ is in $C, \tau f(\lambda)=f\left(\phi^{-1} \gamma(\lambda)\right)$. Clearly $\tau$ is an isomorphism of $C$ onto itself. Further $\tau \phi(\lambda)=\gamma(\lambda)$ and so $\tau B$, the image of $B$ under $\tau$, includes $\gamma(\lambda)$. Since polynomials in $\gamma(\lambda)$ are dense in $A, \tau B$ includes $A$. Let now $B^{\prime}$ include $B$. Then $\tau B^{\prime}$ includes $\tau B$ and hence $\tau B^{\prime}$ includes $A$. By Theorem 1, then, either $\tau B^{\prime}=C$ or $\tau B^{\prime}=A$. If $\tau B^{\prime}=C$, then $B^{\prime} \equiv C$, since $\tau$ is an automorphism. If $\tau B^{\prime}=A$, then $\tau B^{\prime}$ is included in $\tau B$ and so $\tau B^{\prime}=\tau B$. Hence $B^{\prime}=B$. q.e.d.

Theorem 1 is a special case of Theorem 2 , since $A$ contains the function $\phi(\lambda)=\lambda$. As a corollary of Theorem 1, we also obtain the following result due to Rudin [5]: Let $M$ be an algebra of functions continuous in the closed disk $|z| \leqq 1$. Suppose every $F$ in $M$ attains the maximum of its modulus on the boundary $|z|=1$. Then if one schlicht analytic function belongs to $M$, every $F$ in $M$ is analytic in $|z|<1$.

Proof. Let $\bar{M}$ be the closure of $M$ in the sense of uniform approximation on $|z| \leqq 1$. Since $M$ contains one schlicht function, $\bar{M}$ contains all analytic functions continuous in $|z| \leqq 1$. Also $\bar{M}$ is an algebra and if $F$ is in $\bar{M},|F(z)| \leqq \sup _{|\lambda|=1}|F(\lambda)|$ for all $z,|z| \leqq 1$.

Let $A^{\prime}$ be the restriction of $\bar{M}$ to $|z|=1$. Then $A^{\prime}$ is a subalgebra of $C$ and $A^{\prime}$ is closed since $\bar{M}$ is closed and also for any sequence $f_{n}$ in $A^{\prime}, \sup _{|z| \leqq 1}\left|f_{n}(z)-f_{m}(z)\right| \leqq \sup _{|\lambda|-1}\left|f_{n}(\lambda)-f_{m}(\lambda)\right|$. Suppose $A^{\prime}$ $=C$. Then the functional which assigns to each $f$ in $C$ the number $F(0)$, where $F$ is the unique function in $\bar{M}$ with boundary value $f$, is a multiplicative functional on $C$ and so must equal $f\left(\lambda_{0}\right)$ for some $\lambda_{0},\left|\lambda_{0}\right|=1$. Thus for all $F$ in $\bar{M}, F(0)=F\left(\lambda_{0}\right)$, which is false since $F(\lambda)=\lambda$ is in $\bar{M}$. Thus $A^{\prime} \neq=C$.

Now $A^{\prime}$ includes $A$, since $\bar{M}$ contains all analytic functions continuous in $|z| \leqq 1$. By Theorem 1 , then, $A^{\prime}=A$, and so if $F$ is in $\bar{M}$ there exists $F_{1}$ analytic with $F_{1}(\lambda)=F(\lambda)$ if $|\lambda|=1$. Since $F_{1}$ belongs to $\bar{M}$, it follows that $F_{1}(z)=F(z)$ for all $z,|z| \leqq 1$. q.e.d.

\section{REFERENCES}

1. Z. L. Leibenson, On the ring of continuous functions on a circle, Uspehi Matem. Nauk N.S. vol. 7 (1952) pp. 163-164.

2. C. Esseen, Fourier analysis of distribution functions, Acta Math. vols. 77-78 (1945-46) pp. 3-125.

3. A. Zygmund, Trigonometrical series, Chelsea, 1952.

4. N. N. Lusin and I. I. Priwaloff, Sur l'unicité et multiplicité des fonctions ana- 
lytiques, Ann. Ecole Norm. vol. 42 (1925).

5. W. Rudin, Analyticity and the maximum modulus principle, Bull. Amer. Math. Soc. Abstract 58-6-655.

YALE UNIVERSITY

\section{SOLUTION OF BERNSTEIN'S APPROXIMATION PROBLEM ${ }^{1}$}

\section{HARRY POLLARD}

In his famous monograph on approximation theory [2], S. Bernstein initiated the study of the closure properties of sets of functions $\left\{u^{n} K(u)\right\}_{0}^{\infty}$ on the real line. It is supposed that $K(u)$ is continuous on $(-\infty, \infty)$ and that $u^{n} K(u)$ vanishes at $u= \pm \infty$ for each value of $n$. The problem is to decide when the set $\left\{u^{n} K(u)\right\}$ is fundamental in the space $C_{0}$ of functions continuous on $(-\infty, \infty)$, vanishing at $\pm \infty$, and normed by $\|f\|=\max |f(u)|$. So far no necessary and suffcient conditions have been given. A recent paper of Carleson [3] reviews most of the known results, but the paper [1] which seems to come closest to the true conditions has been overlooked.

It is the purpose of this note to give a complete solution. It applies to either real- or complex-valued functions and may be read either way.

THEOREM. In order that $\left\{u^{n} K(u)\right\}_{0}^{\infty}$ be fundamental in $C_{0}$ it is necessary and sufficient that

$$
\begin{gathered}
K(u) \neq 0, \quad-\infty<u<\infty ; \\
\int_{-\infty}^{\infty} \frac{\log |K(u)|}{1+u^{2}} d u=-\infty ;
\end{gathered}
$$

and that there exists a sequence of polynomials $p_{n}$ such that

$$
\lim _{n \rightarrow \infty} p_{n}(u) K(u)=1 ; \quad\left|p_{n}(u) K(u)\right| \leqq C, \quad-\infty<u<\infty .
$$

1. The necessity. The necessity of (1) is obvious and of (2) is well known $[1 ; 3]$. To prove the necessity of the remaining conditions let $0_{n}(u)$ denote the continuous function which is unity on $(-n, n)$, vanishes outside $(-n-1, n+1)$, and is linear in the remaining in-

Presented to the Society, April 25, 1953; received by the editors April 2, 1953.

${ }^{1}$ Research supported in part by a grant from the Office of Naval Research. 